\title{
Vibronic Dynamics of the Ultrafast all-trans to 13-cis Photoisomerization of Retinal in Channelrhodopsin-1
}

\author{
Christoph Schnedermann, ${ }^{\dagger, \mathrm{a}}$ Vera Muders, ${ }^{\ddagger, a}$ David Ehrenberg, ${ }^{\S}$ Ramona Schlesinger, ${ }^{\ddagger}$ \\ Philipp Kukura, ${ }^{*},{ }^{\dagger}$ Joachim Heberle*,\$ \\ † Physical and Theoretical Chemistry Laboratory, University of Oxford, South Parks Road, Oxford OX1 3QZ, U.K. \\ * Genetic Biophysics, Department of Physics, and ${ }^{\S}$ Experimental Molecular Biophysics, Department of Physics, Freie \\ Universität Berlin, Arnimallee 14, 14195 Berlin, Germany \\ ${ }^{\mathrm{a}}$ These authors contributed equally to this work
}

\section{Sample Preparation}

$\mathrm{CaChR} 1$ was cloned, expressed and purified as described previously. ${ }^{1}$ An additional gel filtration step was introduced to yield highly purified sample with a ratio of $\mathrm{OD}_{280 \mathrm{~nm}} / \mathrm{OD}_{518 \mathrm{~nm}}=2.5$. The protein was concentrated to $10 \mathrm{mg} / \mathrm{mL}$ in an aqueous solution of $100 \mathrm{mM} \mathrm{NaCl}, 20 \mathrm{mM}$ Hepes, $\mathrm{pH} 7.4$ with $0.03 \%$ dodecylmaltoside. The sample was kept in the dark prior to the experiments.

\section{Ultrafast Spectroscopy}

The experimental setup has been described in detail elsewhere. ${ }^{2}$ Briefly, femtosecond pulses were provided by a Pharos $6 \mathrm{~W}$ amplifier system $(1030 \mathrm{~nm}, 200 \mathrm{fs}, 5.4 \mathrm{~W}, 2 \mathrm{kHz})$. Broadband probe pulses $(500-900 \mathrm{~nm}$, $2 \mathrm{~nJ}$, transform-limit $4.5 \mathrm{fs}$ ) were obtained by focussing $3 \mu \mathrm{J}$ of fundamental into a $3 \mathrm{~mm}$ sapphire crystal and detected using a home-built single-shot prism spectrograph equipped with a CMOS array detector (LW-ELIS-1024A-1394). Frequency-doubling of the fundamental generated excitation pulses $(515 \mathrm{~nm}$, $200 \mathrm{fs}$ ) employed in the BB-IVS experiment while ultrashort pump pulses in the visible $(560 \mathrm{~nm}, 8 \mathrm{fs})$ and near-IR ( $800 \mathrm{~nm}, 9 \mathrm{fs}$ ) were produced as described previously. ${ }^{3}$ All experiments were carried out using an $\mathrm{OD}_{518 \mathrm{~nm}}=10$ sample flowed through a $200 \mu \mathrm{m}$ flowcell at a flow speed sufficient to ensure a fresh sample volume for every shot employing parallel polarized pulses with diameters of $34 \mu \mathrm{m}$ (probe), $75 \mu \mathrm{m}$ (530 $\mathrm{nm}$ short visible pump, $150 \mathrm{~nJ}), 65 \mu \mathrm{m}$ (near-IR, $160 \mathrm{~nJ})$ and $90 \mu \mathrm{m}(515 \mathrm{~nm}, 50 \mathrm{~nJ})$ at the sample.

Error margins for the evaluated time constants were obtained from the covariance matrix of the global fit after applying a second-order Savitzki-Golay filter ${ }^{4}$ to the transient absorption data in order to reduce the effective time resolution to $50 \mathrm{fs}$. The frequency resolution of the extracted IVS Raman spectra was $\sim 15$ $\mathrm{cm}^{-1}$. 


\section{Wavelength Dependence of Transient Absorption Dynamics}

The photodynamics of channelrhodopsin-1 were recently found to be pump wavelength dependent. Red excitation wavelengths $(\sim 550 \mathrm{~nm})$ are associated with a primary excitation of all-trans retinal chromophores, while blue excitation wavelengths $(\sim 500 \mathrm{~nm})$ lead additionally to photoexcitation of ground-state 13-cis retinal chromophores. To support the transient absorption (TA) data obtained with a pump pulse centered at $560 \mathrm{~nm}(8 \mathrm{fs})$ covering the entire red edge of the absorption spectrum (Figure 1), we carried out TA measurements with pump pulses ranging from $530-590 \mathrm{~nm}$ with reduced pulse durations of $16 \mathrm{fs}$ (Figure S1, bottom). By comparing selected transients of the ground-state bleach $(517 \mathrm{~nm})$ and stimulated emission band ( $826 \mathrm{~nm})$, we find no differences in the overall dynamics as we scan the pump pulse center wavelength across the red edge of the absorption region (Figure S1, top). Minor differences occur, however, in the photoproduct region at $596 \mathrm{~nm}$ after a time delay of $500 \mathrm{fs}$. We attribute this observation to a decrease in the available energy after photoexcitation, which results in faster vibrational relaxation on the $P_{1}$ potential energy surface. This process affects the second time constant ( $\left.\sim 580 \mathrm{fs}\right)$ but the initial dynamics of the process remain largely unchanged, verifying that the red edge of the absorption spectrum is predominantly associated with all-trans retinal chromophores which photoisomerize with a time constant of $\sim 110$ fs. The structural information derived from the TA results employing a $560 \mathrm{~nm}$ ( 8 fs) pump pulse (Figure 2) are thus representative of the photoisomerization reaction of all-trans retinal in channelrhodopsin-1.
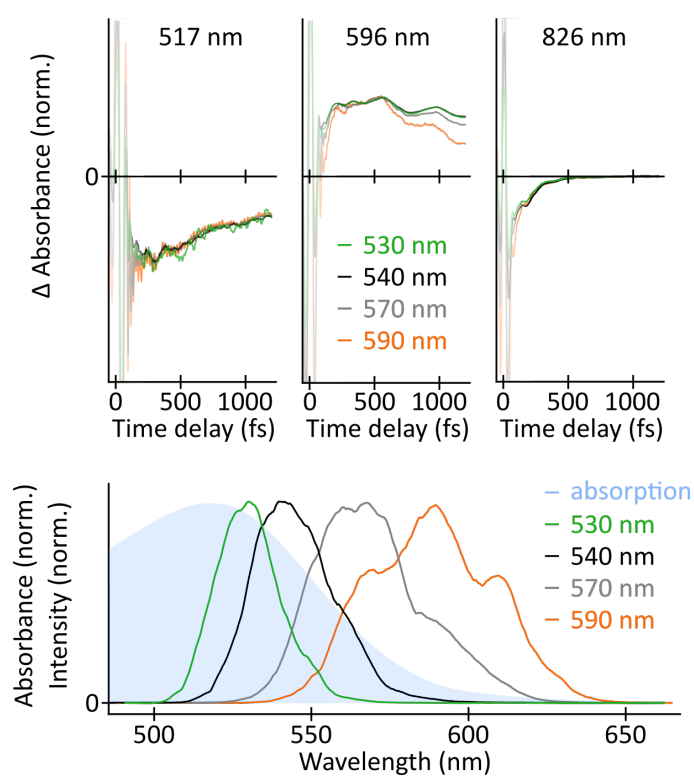

Figure S1. Pump wavelength dependence of transient absorption dynamics. Top: Selected transients representing the ground-state bleach $(517 \mathrm{~nm})$, photoinduced absorption $(596 \mathrm{~nm})$ and stimulated emission $(826$ $\mathrm{nm}$ ) as a function of pump wavelength (colored transients). Early time delays are dominated by coherent artifact contributions and have been shaded for visibility purposes. Bottom: Absorption spectrum (blue) and pump pulse spectra centered at indicated wavelengths. All pulses were compressed to a near transform-limited duration of 16 fs. 


\section{Broadband Impulsive Vibrational Spectroscopy (BB-IVS)}

BB-IVS probes vibrational coherence (VC) generated with a short impulsive pump pulse as a function of impulsive pump-probe delay. The addition of an actinic pump pulse prior to the impulsive pump pulse leads to two different sets of VC: (1) ground-state only (actinic OFF) and (2) a mixture of reactant and photoproduct VC (actinic ON). By subtracting the VC associated with actinic OFF and actinic ON in the time domain we retrieve the VC of the photoproduct (OFF-ON), as outlined in detail elsewhere. ${ }^{2}$ Fourier transformation retrieves the spectral content of the VC.

The Fourier power spectra 1 ps after photoexcitation for channelrhodopsin-1 are displayed in Figure S2. We find that the actinic OFF and actinic ON spectra are dominated by ground-state features at 1013,1162 and $1531 \mathrm{~cm}^{-1}$ which exhibit a reduction in intensity due to the actinic pump pulse. In contrast, modes at $860,961,1196$ and $1301 \mathrm{~cm}^{-1}$ show an increase in intensity if the actinic pump pulse is present. The appearance of new bands in the actinic ON spectrum signifies that a new molecular species is probed. Application of a subtraction algorithm ${ }^{2}$ retrieves the prominent $\mathrm{P}_{1}$ bands while significantly reducing the ground-state bands, as seen e.g. at $1013 \mathrm{~cm}^{-1}$.

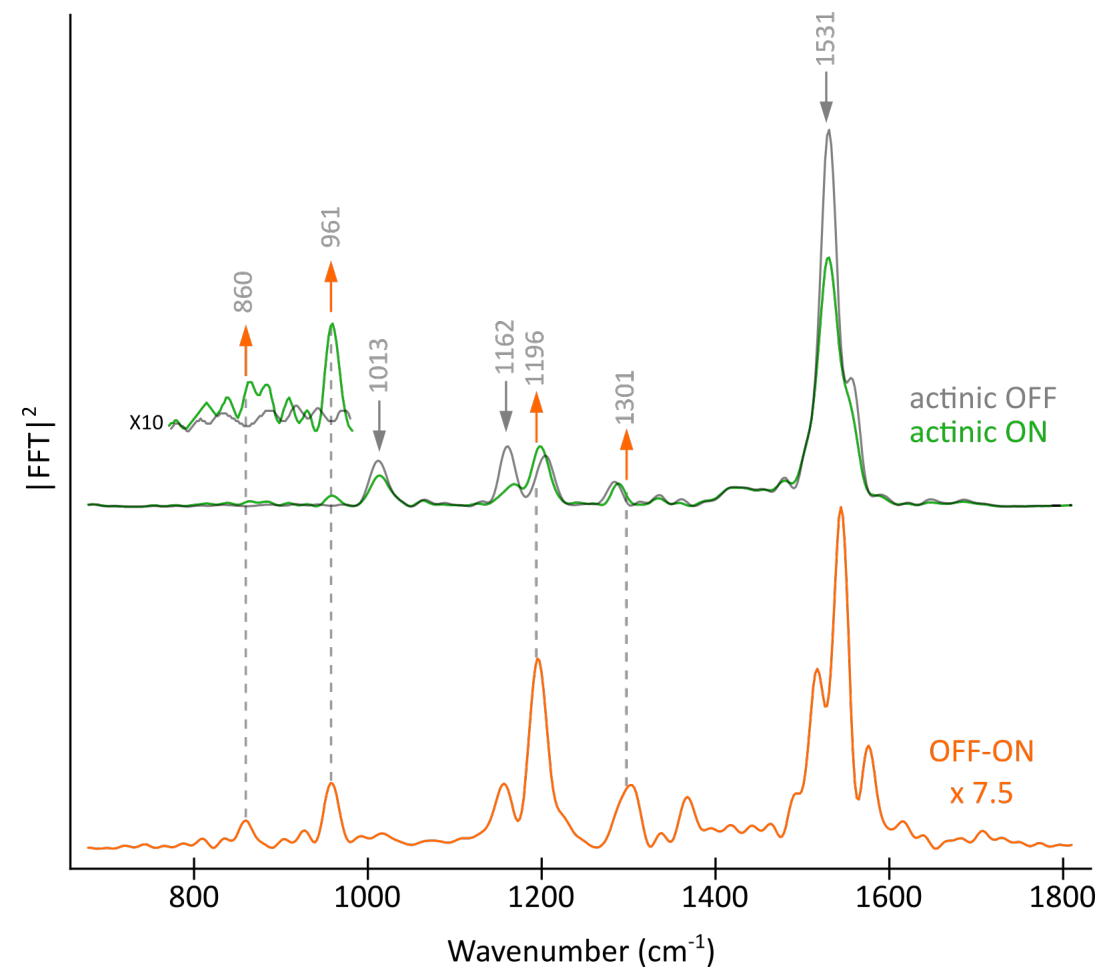

Figure S2. Comparison of BB-IVS spectra at 1 ps actinic-impulsive pump delay. Top: Raw Fourier power spectra averaged from 620-680 $\mathrm{nm}$ in the absence (gray, OFF) and presence (green, ON) of the actinic pump pulse. Bottom: Retrieved Fourier power spectrum after subtraction as outlined elsewhere. ${ }^{2}$ Photoexcitation at $515 \mathrm{~nm}$ (200 fs) was followed after $1 \mathrm{ps}$ by an impulsive pump pulse at $800 \mathrm{~nm}$ ( $9 \mathrm{fs})$. Vertical dashed lines indicate important $\mathrm{P}_{1}$ marker bands and are associated with an increase in the actinic ON spectrum (orange arrows). Ground-state bands are reduced in intensity by the actinic pump pulse denoted by gray arrows. All spectra were corrected for the finite time resolution of the time-domain experiment. ${ }^{5}$ 


\section{Resonance Raman Spectroscopy at Cryogenic Temperature}

$5 \mu \mathrm{L}$ concentrated $\mathrm{CaChR} 1$ sample $(5-10 \mathrm{mg} / \mathrm{mL}$ in $5 \mathrm{mM} \mathrm{NaCl}, 5 \mathrm{mM}$ Hepes, $\mathrm{pH} 7.4,0.05 \%$ dodecylmaltoside) was dried on top of a quartz crucible and rehydrated by vapour diffusion of $3 \mu \mathrm{L}$ of a water/glycerol mixture $(9 / 1 \mathrm{w} / \mathrm{w})$ placed nearby the protein film. The sample was cooled down to $80 \mathrm{~K}$ with a cryostat (freezing stage TMHS600, Linkam). For the resonance Raman experiment on ground-state $\mathrm{CaChR1}$, the emission line at $647 \mathrm{~nm}$ of a Krypton ion laser (Innova 90C, Coherent) was used (see ${ }^{6,7}$ for more details on the Raman setup). The spectral resolution of the Raman spectra was $3 \mathrm{~cm}^{-1}$. Raman spectra were recorded in the presence and absence of the photo-activating laser $\left(\mathrm{Ar}^{+}\right.$laser from Melles Griot with emission at $514 \mathrm{~nm}$ and an intensity of $60 \mathrm{~mW}$ ), under otherwise identical conditions.

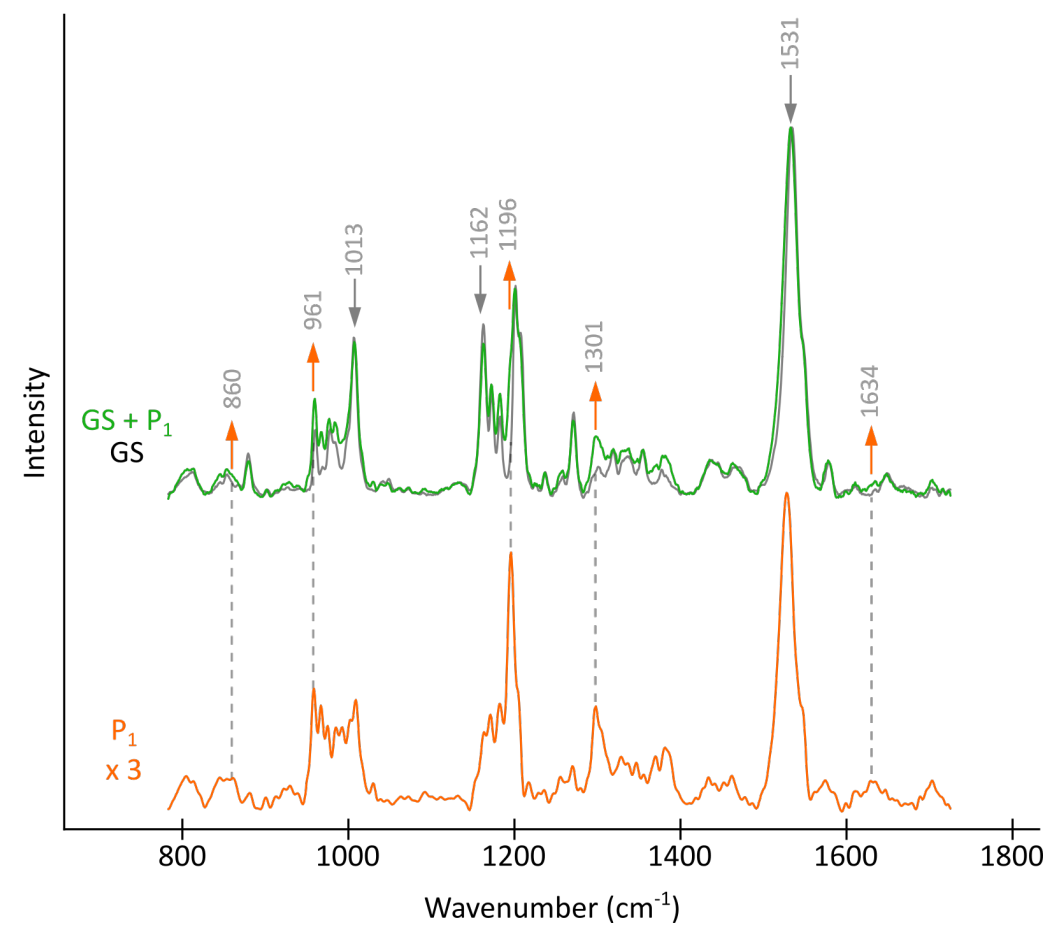

Figure S3. Comparison of Raman spectra of $C a C h R 1$ at $80 \mathrm{~K}$. Top: Resonance Raman spectra of the ground state (GS, gray) and the photostationary mixture (GS $+\mathrm{P}_{1}$, green). Bottom: Resonance Raman spectrum of the $\mathrm{P}_{1}$ intermediate (orange). Illumination at $514 \mathrm{~nm}$ prepared the photostationary mixture which was investigated under pre-resonant conditions at $647 \mathrm{~nm}$. Vertical dashed lines indicate important $\mathrm{P}_{1}$ marker bands and are associated with an increase in the GS $+\mathrm{P}_{1}$ spectrum (orange arrows). Ground-state bands are reduced in intensity in the GS $+\mathrm{P}_{1}$ spectrum denoted by gray arrows.

During illumination with $514 \mathrm{~nm}$, a band at $1196 \mathrm{~cm}^{-1}$ emerges from the Raman spectrum of the photostationary state (Figure S3). This retinal fingerprint band is indicative for the occurrence of 13-cis retinal, whereas the band at $1201 \mathrm{~cm}^{-1}$ is characteristic of the all-trans configuration. To extract the $\mathrm{P}_{1}$ spectrum from the photo-stationary mixture, the contribution of ground-state $\mathrm{CaChR} 1$ was carefully subtracted by choosing an appropriate scaling factor. Considering the ratio of the band at 1196 to $1201 \mathrm{~cm}^{-1}$ yields a $40 \%$ spectral contribution of the $\mathrm{P}_{1}$ intermediate in the photo-stationary mixture. To take spectral overlap and inaccuracies in baseline correction into account, we employed a subtraction method based on asymmetric 
least squares that was introduced by Yang et al. ${ }^{8}$ Using the conservation of molecules constraint ${ }^{9}$ a fraction of $25 \%$ of $C a C h R 1$ was determined that is photo-converted to the $\mathrm{P}_{1}$ intermediate.

\section{Comparison of Vibrational Difference Spectra}

The FT-IR difference spectrum of the $\mathrm{P}_{1}$ intermediate at $80 \mathrm{~K}$ has recently been published. ${ }^{10}$ In order to compare this spectrum with the observed Raman data, the Raman spectrum of the ground state was subtracted from the $\mathrm{P}_{1}$ spectrum. The scaling was chosen to match the difference intensities of the FT-IR spectrum (Figure S4). Negative bands correspond to ground-state vibrations while positive bands are due to vibrational motion of the $\mathrm{P}_{1}$ intermediate. Overall, the Raman and the FT-IR data are in excellent agreement confirming that the same $\mathrm{P}_{1}$ intermediate has been trapped at $80 \mathrm{~K}$. The bands at 1196 and 1301 $\mathrm{cm}^{-1}$ are strongly pronounced in both difference spectra indicating a 13-cis configuration of the retinal in $\mathrm{P}_{1}$. The band at $1634 \mathrm{~cm}^{-1}$ has been assigned to the protonated Schiff base vibration. ${ }^{10}$ This assignment is supported by the Raman data confirming a $\mathrm{P}_{1}$ intermediate with 13-cis retinal and a protonated Schiff base. The high similarity of the vibrational differences recorded by resonance Raman scattering and IR absorption confirm that the initial photoreaction is restricted to the chromophore retinal and its immediate environment.

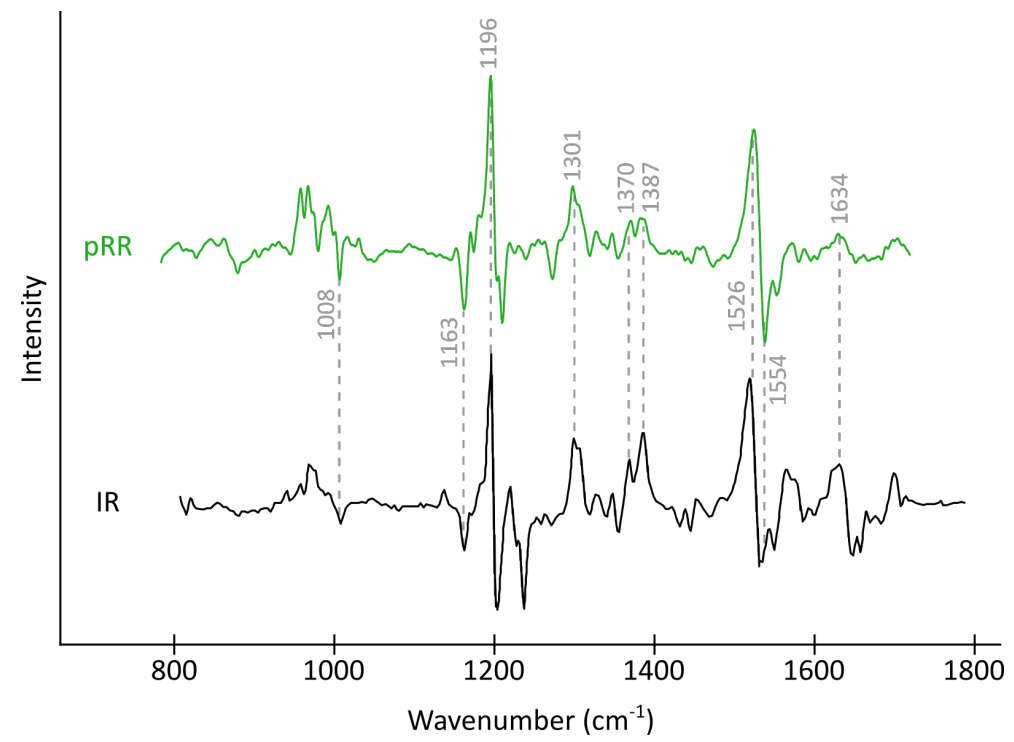

Figure S4. Comparison of the difference spectra obtained by pre-resonance Raman (pRR, green) and FTIR spectroscopy (IR, black) on $\boldsymbol{C a C h R 1}$ at $80 \mathrm{~K}$. Raman spectra were subtracted to match the difference intensities of the FT-IR spectrum. Similar frequencies are indicated in gray. Note the consistent bands for the protonated Schiff base $\left(1634 \mathrm{~cm}^{-1}\right)$ and the marker bands for the 13-cis configuration of retinal at 1196 and 1301 $\mathrm{cm}^{-1}$. The FT-IR difference spectrum was reproduced with permission from the American Chemical Society. ${ }^{10}$ 


\section{Comparison of Transient Dynamics in Different Rhodopsins}

To understand the transient absorption dynamics found for $C a \mathrm{ChR} 1$, it is instructive to compare to the dynamics in visual rhodopsin (Rho) and bacteriorhodopsin (bR) as shown in Figure S5. In the case of Rho, the excited-state potential is barrierless. The characteristic transient feature is the disappearance of a stimulated emission band in the near-IR and immediate reappearance of a photoproduct absorption together with extensive red shifting of the stimulated emission and blue-shifting of the photoproduct absorption. $^{11,12}$ Since we do not observe such a characteristic spectral change, we believe that there must be a barrier on the excited state. This conclusion is partially fueled by respective observations for bR, where the $500 \mathrm{fs}$ excited-state lifetime is attributed to an energetic excited state barrier and the spectral resemblance of the transient dynamics to $C a \mathrm{ChR} 1$ is excellent. ${ }^{13,14}$ We therefore propose a relatively shallow excited-state barrier to represent that $\mathrm{CaChR} 1$ is more similar to bR than to Rho in terms of dynamics. The barrier is most likely of dynamic origin due to the fast time constant of $110 \mathrm{fs}{ }^{15}$
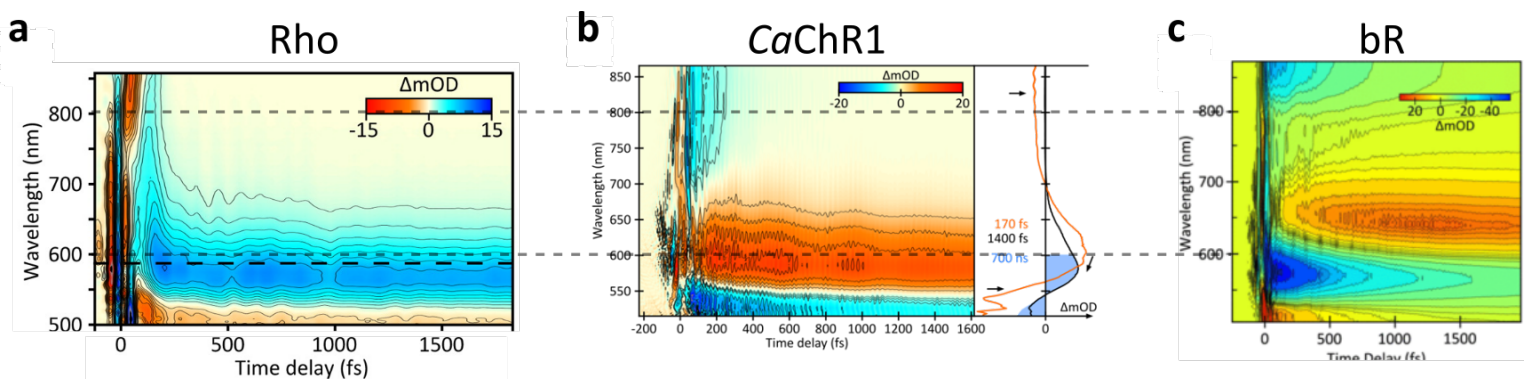

Figure S5. Comparison of transient absorption dynamics between (a) rhodopsin (Rho), (b) channelrhodopsin-1 ( $\mathrm{CaChR1)}$ and (c) bacteriorhodopsin (bR). CaChR1 does not show the characteristic conical intersection (CI) dynamics found in the barrierless photoisomerization of Rho, but forms its first photointermediate in a much shorter time than bR. This is indicative of a dynamic barrier due to a shallow excited-state plateau for $C a \mathrm{ChR} 1$. Dashed horizontal lines are a guide to the eye for matching probe wavelengths. The data for Rho was reprinted with permission from Schnedermann et al, J. Am. Chem. Soc. 2015, 137 (8), pp 2886-91, copyright 2015, American Chemical Society. ${ }^{11}$ The data for bR was reprinted with permission from Liebel et al., Phys. Rev. Lett. 2014, 112, 38301146, copyright (2014) by the American Physical Society. ${ }^{14}$

\section{Linear Prediction Singular Value Decomposition Analysis of Low- Frequency Modes}

To investigate the phase behavior of the low-frequency coherences as a function of probe wavelength, we analyzed the residual coherences with linear prediction singular value decomposition (LPSVD) as demonstrated by Barkhuijsen and Ormondt. ${ }^{16,17}$ LPSVD is used to obtain the frequencies and lifetimes of the oscillatory signal followed by a linear fitting routine to extract reliable amplitude and phase values at each probe wavelength. To describe the residual coherent oscillations observed in $\mathrm{CaChR} 1$, we employ 12 exponentially decaying sinusoidal oscillations resulting in a fit-residual equal to the experimental noise floor (Figure S6a). To illustrate the approach, we restrict our analysis to the dominant mode at $\sim 80 \mathrm{~cm}^{-1} \mathrm{by}$ removing all other oscillations from the coherence signal, as demonstrated in Figure S6b. Subsequently, 
we obtain frequency, lifetime, amplitude and phase values for this mode at each probe wavelength across the photoproduct absorption from 550-650 nm (Figure S6c).

Based on this analysis we can re-evaluate the frequency of the dominant low-frequency mode to be 86.4 $( \pm 1.0) \mathrm{cm}^{-1}$ with a lifetime of $1.19( \pm 0.17) \mathrm{ps}$, a constant phase of $1.77( \pm 0.05) \pi$ and a wavelengthresolved oscillatory amplitude profile in excellent agreement with the photoproduct absorption spectrum. The constant phase of $1.77 \pi$ indicates an impulsively driven mode and the lifetime of 1.19 ps suggests that this mode corresponds to the photoproduct state, rather than a (potentially) overlapping excited state, found to have a much shorter lifetime (110 fs).

In principle, it is possible that the observed oscillation arises from impulsive excitation of an strongly inhomogenously broadened ground-state vibrational coherence which the analysis is not revealing due to our limited probe range in the blue $(<500 \mathrm{~nm})$. The off-resonant Fourier spectrum (Figure $2 \mathrm{~d}$ ) does indeed show a band at $\sim 90 \mathrm{~cm}^{-1}$, which could match such an assignment, although the corresponding intensity appears much too weak to warrant such an assignment. We thus tentatively assign the $86 \mathrm{~cm}^{-1}$ mode to be a photoproduct mode, in agreement with the observations for visual rhodopsin. ${ }^{18} \mathrm{~A}$ more targeted investigation probing all electronic resonances with longer time delays and more controlled pulse parameters will have to be conducted to reveal conclusively the origin of these low-frequency modes.

a
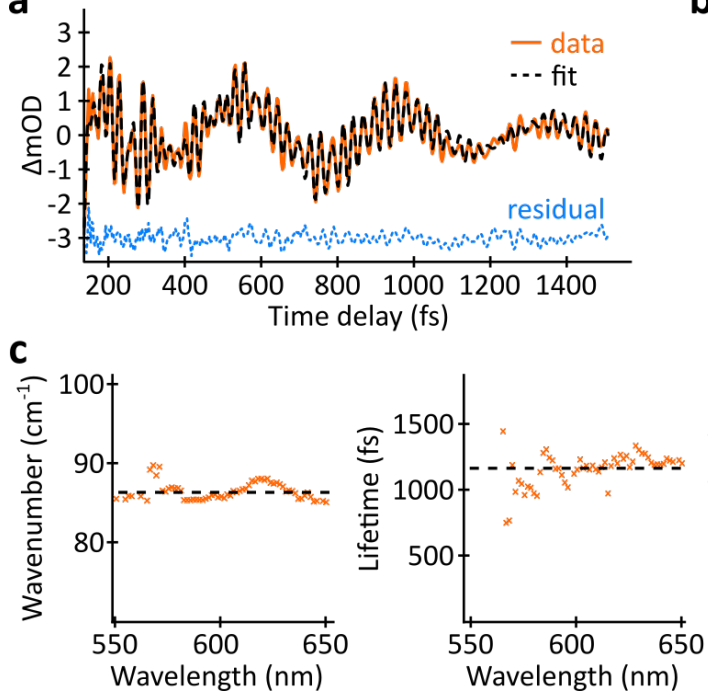
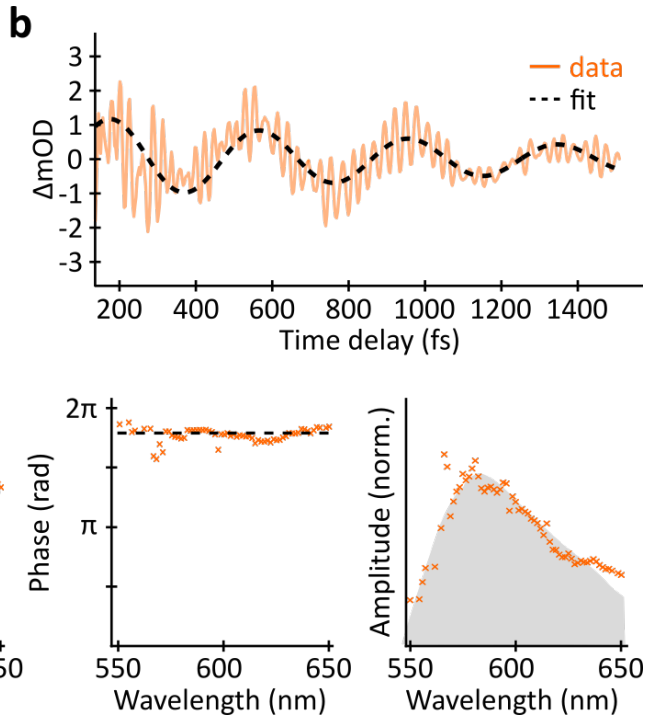

Figure S6. Linear prediction singular value decomposition analysis of the low-frequency region. (a) coherent oscillation at $598 \mathrm{~nm}$ (orange) described by 12 exponentially decaying oscillations (black, dashed) resulting in a noise-limited residual (blue). (b) Retrieved fit to a single mode at $\sim 86 \mathrm{~cm}^{-1}$. (c) Wavelengthresolved frequency, lifetime, phase and amplitude profiles of the dominant low-frequency mode at $86 \mathrm{~cm}^{-1}$. The grey shaded area in the amplitude graph (right) represents the $\mathrm{P}_{1}$ absorption spectrum. 


\section{References}

(1) Muders, V.; Kerruth, S.; Lórenz-Fonfría, V. A.; Bamann, C.; Heberle, J.; Schlesinger, R. FEBS Lett. 2014, 588 (14), 2301.

(2) Liebel, M.; Kukura, P. J. Phys. Chem. Lett. 2013, 4 (8), 1358.

(3) Liebel, M.; Schnedermann, C.; Kukura, P. Opt. Lett. 2014, 39 (14), 4112.

(4) Savitzky, A.; Golay, M. J. E. Anal. Chem. 1964, 36 (8), 1627.

(5) Liebel, M.; Schnedermann, C.; Wende, T.; Kukura, P. J. Phys. Chem. A 2015, 119 (36), 9506.

(6) Radu, I.; Bamann, C.; Nack, M.; Nagel, G.; Bamberg, E.; Heberle, J. J. Am. Chem. Soc. 2009, 131 (21), 7313.

(7) Nack, M.; Radu, I.; Bamann, C.; Bamberg, E.; Heberle, J. FEBS Lett. 2009, 583 (22), 3676.

(8) Yang, C.; Peng, S.; Xie, Q.; Peng, J.; Wei, J.; Hu, Y. In 2011 4th International Conference on Biomedical Engineering and Informatics (BMEI); IEEE, 2011; pp 1724-1728.

(9) Ames, J. B.; Raap, J.; Lugtenburg, J.; Mathies, R. A. Biochemistry 1992, 31 (50), 12546.

(10) Ogren, J. I.; Yi, A.; Mamaev, S.; Li, H.; Lugtenburg, J.; DeGrip, W. J.; Spudich, J. L.; Rothschild, K. J. Biochemistry 2015, 54 (2), 377.

(11) Schnedermann, C.; Liebel, M.; Kukura, P. J. Am. Chem. Soc. 2015, 137 (8), 2886.

(12) Polli, D.; Altoè, P.; Weingart, O.; Spillane, K. M.; Manzoni, C.; Brida, D.; Tomasello, G.; Orlandi, G.; Kukura, P.; Mathies, R. A; Garavelli, M.; Cerullo, G. Nature 2010, 467 (7314), 440.

(13) Mathies, R. A.; Brito Cruz, C.; Pollard, W. T.; Shank, C. V. Science. 1988, 240 (4853), 777.

(14) Liebel, M.; Schnedermann, C.; Bassolino, G.; Taylor, G.; Watts, A.; Kukura, P. Phys. Rev. Lett. 2014, 112 (23), 238301.

(15) Dokukina, I.; Weingart, O. Phys. Chem. Chem. Phys. 2015, 17 (38), 25142.

(16) Barkhuijsen, H.; de Beer, R.; Bovée, W. M. M.; van Ormondt, D. J. Magn. Reson. 1985, 61 (3), 465.

(17) Barkhuijsen, H.; de Beer, R.; van Ormondt, D. J. Magn. Reson. 1987, 73 (3), 553.

(18) Wang, Q.; Schoenlein, R. W.; Peteanu, L. A.; Mathies, R. A.; Shank, C. V. Science. 1994, 266 (5184), 422. 